\title{
Continuous Subcutaneous Administration of Miso Extracts Attenuates Salt-Induced Hypertension in Dahl Salt-Sensitive Rats
}

\author{
Namiko Shimizu, Dong Dong Du, Hiroe Sakuyama, Yusai Ito, Masaru Sonoda, \\ Kiyoshi Kawakubo, Yoshio Uehara* \\ Division of Clinical Nutrition, Faculty of Home Economics, Kyoritsu Women's University, Tokyo, Japan \\ Email: *yuehara@kyoritsu-wu.ac.jp
}

Received 9 March 2015; accepted 21 May 2015; published 26 May 2015

Copyright (C) 2015 by authors and Scientific Research Publishing Inc.

This work is licensed under the Creative Commons Attribution International License (CC BY).

http://creativecommons.org/licenses/by/4.0/

(c) (i) Open Access

\section{Abstract}

Objective: Long-term intake of Miso attenuates hypertension in Dahl salt-sensitive (Dahl S) rats through an increased urinary sodium excretion. We examined whether a bolus injection into the peritoneal cavity (i.p.) or a continuous subcutaneous infusion of a Miso extract attenuates hypertension in Dahl $S$ rats. Materials and Methods: We investigated the effects of a bolus, i.p. injection of $50 \mathrm{mg}$ Miso extract in $0.5 \mathrm{~mL}$ on hypertension in Dahl $\mathrm{S}$ rats, and examined whether a long-term subcutaneous infusion of the Miso extract $(50 \mathrm{mg}$ Miso/day), using an osmotic mini-pump working for 14 days, attenuates hypertension in Dahl $S$ rats. Results: A bolus, i.p. injection of 50 mg Miso extract decreased SBP in 2 hrs. The reduction was significant at $4 \mathrm{hrs}$, and SBP returned to the baseline at $24 \mathrm{hrs}$. The SBP reduction at $4 \mathrm{hrs}$ after the injection was increasingly greater each day during the 4 days. The SBP reduction by the Miso extract was dose-dependent and the antihypertensive activity occurs in a $<5 \mathrm{kDa}$ fraction of the extract. The subcutaneous infusion of $50 \mathrm{mg}$ Miso extract/day for 14 days significantly attenuated hypertension in Dahl $S$ rats. The SBP reduction was associated with significant decreases in the heart and kidney weights. Urinary protein excretion significantly decreased in the Miso group. The SBP reduction was not associated with increases in either urinary sodium excretion or fractional excretion of sodium. Conclusions: SBP reduction by very low-dose of the Miso extract may be mediated partly by mechanisms other than renal sodium handling.

\section{Keywords}

Miso, Salt Induced Hypertension, Dahl Rats

\footnotetext{
${ }^{*}$ Corresponding author.
} 


\section{Introduction}

Miso is a traditional Japanese food that was originally described in Taiho Ritsurei in 701 AD. Miso is manufactured using soybeans along with malted rice and salt; a significant amount of salt is needed for complete fermentation. One serving of Miso soup generally contains $1-1.5 \mathrm{~g}$ salt which supports the long-standing belief that traditional Miso soup is to blame for the high incidence of salt-sensitive hypertension and subsequent cerebral stroke in Japan.

Interestingly, however, we and investigators from other laboratory have reported in the previous study that long-term intake of Miso soup or Miso attenuates development of salt-induced hypertension in Dahl saltsensitive (Dahl S) rats when compared with rats given the same amount of saline solutions as the salt contents in Miso soup [1]-[3]. The reduction in blood pressure in the Miso group corresponds to approximately $30 \%$ reduction in salt intake. The mechanisms for the blood pressure reduction are uncertain; however, several hypotheses are proposed. First, Miso inhibits salt absorption in the intestine. Second, the constituent(s) of Miso soup increase urinary excretions of sodium, thereby decreasing salt loading. Third, the functions of Miso constituents are similar to vasodilators.

In our preliminary study using metabolic cages, sodium absorption in the intestine was not influenced by the long-term intake of Miso in normotensive Wistar rats (unpublished data) [4]. In addition, the long-term intake of $5 \%$ Miso enhanced natriuresis and diuresis in association with increased urinary dopamine excretion, suggesting that oral intake of 10 - $20 \mathrm{~g}$ Miso/day attenuates salt-induced hypertension in Dahl S rats through enhanced excretion of sodium in the kidneys [2].

In the present study, we proposed the hypothesis that Miso extract attenuates hypertension through vasodilator effects on vascular walls via the circulation. To test this hypothesis, we injected a Miso extract into the abdominal cavity or subcutaneous tissue and we then examined the blood pressure in Dahl rats with salt-induced hypertension.

\section{Materials and Methods}

\subsection{Acute Effects of Bolus, Intra-Peritoneal Injection of Miso Extract}

\section{Protocol of the study}

We purchased 4-week-old male Dahl salt-sensitive (Dahl S/Iwai) rats from Sankyo Laboratories, Inc., Tokyo, Japan. The Dahl S rats were fed a high-salt $(4 \% \mathrm{NaCl})$ diet to develop salt-induced hypertension. Dahl S rats aged 7 - 8 weeks old were used for assessing the antihypertensive activity of a bolus intra-peritoneal (i.p.) injections of Miso extract.

\section{Manufacture of Miso}

Miso is manufactured from soybeans with malted rice and salt. A significant amount of salt is needed for complete fermentation. One serving of Miso soup contains 1 - $2 \mathrm{~g}$ salt. A representative Japanese meal is composed of rice, the main dish, several side-dishes, e.g., vegetables, etc., and Miso soup. Therefore, when Miso soup is served 3 times a day, $3 \mathrm{~g}$ of salt or more is consumed in addition to the usual salt intake from other foods.

\section{Preparation of Miso extract}

Miso utilized in the present study was offered by the Central Miso Research Institute (Tokyo, Japan). One thousand kilogram Miso is regularly made of $293 \mathrm{~kg}$ soybeans, $144 \mathrm{~kg}$ rice (malted), $130 \mathrm{~kg} \mathrm{NaCl}$, and water (Kome Miso full-boiled and colored light-yellow). Miso was fermented for 3 months [2]. We dissolved $100 \mathrm{~g}$ of Miso in $1000 \mathrm{~mL}$ of water and homogenized the mixture with an ultrasonic POLYTRON (KINEMATICA $\mathrm{GmbH}$, Luzern, Switzerland) blender for $5 \mathrm{~min}$ [1] [2]. The homogenate was autoclaved at $120^{\circ} \mathrm{C}$ for $40 \mathrm{~min}$ and centrifuged at $1.5 \times 10^{4} \mathrm{~g}$ for $30 \mathrm{~min}$. The supernatant was passed through Millipore membranes $(0.25 \mu \mathrm{m}$ pore size) for sterilization. The salt concentration of the Miso extract was $1.3 \%(\mathrm{w} / \mathrm{v})$. We prepared newly the Miso extract every time we tested the antihypertensive activity.

\section{Assay of antihypertensive activity}

We injected $0.5 \mathrm{~mL}$ of the extract from 50 or $25 \mathrm{mg}$ Miso into the peritoneal cavity of hypertensive Dahl S rats aged 7 - 8 weeks and administered $0.5 \mathrm{~mL}$ of $1.3 \%$ or $0.65 \%$ saline solutions corresponding to the concentrations of salt in the Miso extract to the control hypertensive rats. The systolic blood pressure (SBP) was determined using the tail-cuff method of Freedman and Freed with a slight modification in the detection system (Natsume Seisakujo Model KN-210-1; Tokyo, Japan) [5]-[7]. 


\subsection{Long-Term Subcutaneous Infusion of the Miso Extract Using an Osmotic Mini-Pump in Rats with Salt-Induced Hypertension}

Sixteen 4-week-old Dahl S rats were fed a high-salt diet (4\% NaCl, w/w) for 4 weeks and six 4-week-old Dahl S rats were fed a low-salt diet $(0.3 \% \mathrm{NaCl}$, w/w) diet for 4 weeks (low-salt DS). The Dahl S rats fed the high-salt diet (4\% $\mathrm{NaCl}, \mathrm{w} / \mathrm{w})$ were divided into 2 groups at the age of 6 weeks; 1$) 8 \mathrm{Dahl} \mathrm{S}$ rats fed the high-salt with sham-operation (DS-high), and 2) 8 Dahl S fed the high-salt with a subcutaneous continuous infusion of the Miso extract (50 mg/day) using an osmotic mini-pump (ALZET model 2ML2; $5.0 \mu \mathrm{L} / \mathrm{h}$ working for 14 days, Cupertino, CA) (DS-miso) [8]. The osmotic mini-pump filled with 5.4\% saline solution was implanted to the sham Dahl S rats (DS-high). The concentration corresponded to that in the Miso extract. Chow and water were available ad libitum throughout the experiment.

At the end of the study, each rat was placed in a metabolic cage for 3 days consecutively, and 24-h urine samples were collected on the last day for testing. Urine samples were stored at $-80^{\circ} \mathrm{C}$ until assayed. A single investigator measured the blood pressure of all animals in a quiet warm room from 13:00 to 16:00. The blood samples and organs of interest were obtained from rats under pentobarbital anesthesia ( $75 \mathrm{mg} / \mathrm{kg}$ body weight).

We used the osmotic mini-pump ALZET model 2ML2 for subcutaneous administration of the Miso extract [8]. The pump was preconditioned in a normal saline solution overnight before the use. This pump continuously infuses $2.0 \mathrm{~mL}$ of the Miso extract solution $(416 \mathrm{mg} / \mathrm{mL})$ at $5.0 \pm 0.75 \mu \mathrm{L} / \mathrm{h}$. Theoretically, this pump is used for 13 to 17 days. The daily dose of the Miso was $50 \pm 7 \mathrm{mg} /$ day. This dose was much lower than the bolus, i.p. injection of the $50 \mathrm{mg}$ Miso extract in the present study and the doses utilized in our previous studies on oral intake of Miso (around $20 \mathrm{~g}$ Miso/day) [1] [2].

\subsection{Determination of Variables}

Plasma and urinary concentrations of creatinine were measured using an autoanalyzer (Model Hitachi 736; Hitachi Co., Ltd., Tokyo, Japan). Concentrations of $\mathrm{Na}$ were determined by an $\mathrm{Na}^{+}$-sensitive electrode and $\mathrm{Na}$ METER TS-999i determination system (Tokyo Chemical Laboratories, Co, Ltd., Tokyo) [1] [2]. Urinary protein concentrations were measured using a protein assay kit (BioRad, Hercules, CA) [6] [7]. Plasma and urinary osmolality were measured by the standard method, i.e., molecular depression of freezing point [2]. Investigators who were blind to the rat assignment determined the various parameters.

\subsection{Statistical Analysis}

All statistical analyses were performed using STATISTICA software (StatSoft, Tulsa, OK). Values were expressed as means \pm SD. Differences were assessed by one-way factorial analysis of variance (ANOVA) followed by the post-hoc least significant difference (LSD) test. p-values less than 0.05 were considered statistically significant.

\subsection{Guidelines for Handling Rats for Experiments}

We followed the guidelines for experimental animal handling, and our study was approved by the Animal Care Committee of the Kyoritsu Women's University (No. 13003, 2013 and No. 14001, 2014). The experiment was conducted in accordance with the guidelines of the National Institutes of Health.

\section{Results}

\subsection{Acute Changes in SBP in Response to I.P. Injection of Miso Extract}

A bolus i.p. injection of $50 \mathrm{mg}$ Miso extract in $0.5 \mathrm{~mL}$ decreased SBP of Dahl S rats after 2 hrs. The reduction was statistically significant at 4 hrs and the SBP was equal to the baseline after 24 hrs (Figure 1).

The bolus injection once a day was continued for 4days. The SBP values were determined at 4 hrs after the injection on day 2 and day 4 (Figure 2). The repeated injection of the Miso extract significantly decreased SBP on day 2 and day 4. The reductions in SBP were increasingly greater as the injections were repeated. Moreover, the repeated injection of Miso extract lowered the nadir SBP, compared with saline injection by $7.9 \%(p<0.05)$ on day-3 and 9.6\% ( $<0.05)$ on day-5. These suggested that an increase in dose of Miso extract attenuates SBP for 24 hrs. 


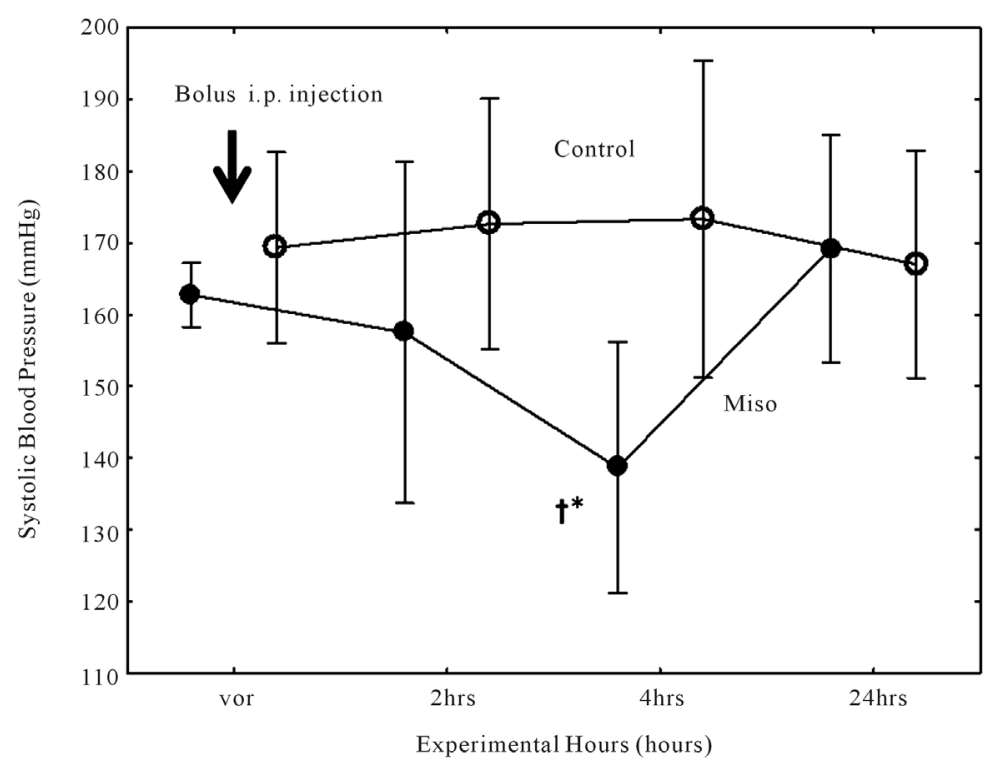

Figure 1. Changes in the SBP in response to a bolus i.p. injection of the Miso extract. Dahl salt-sensitive (Dahl S) rats fed a high-salt diet were given the Miso extract and changes in SBP were noted. The SBP was measured at before the injection (vor), after 2 ( 2 hrs), 4 (4 hrs) and 24 hours (24 hrs). The drawing module avoided the overlay of the SDs bars and the marks were described apart for each test period. Open circle, Dahl S rats given $0.5 \mathrm{~mL} 0.65 \%$ saline solution corresponding to the salt concentration in the Miso extract (Control); closed circle, Dahl S rats given $0.5 \mathrm{~mL}$ from $50 \mathrm{mg}$ Miso (Miso). ${ }^{*} \mathrm{p}<0.05 \mathrm{vs}$ the value in $0.65 \%$ saline solution group, ${ }^{\dagger} \mathrm{p}<0.05$ vs the value before injection of the Miso extract (vor). Group differences were assessed by one-way factorial analysis of variance (ANOVA) followed by a post-hoc least significant difference (LSD) test.

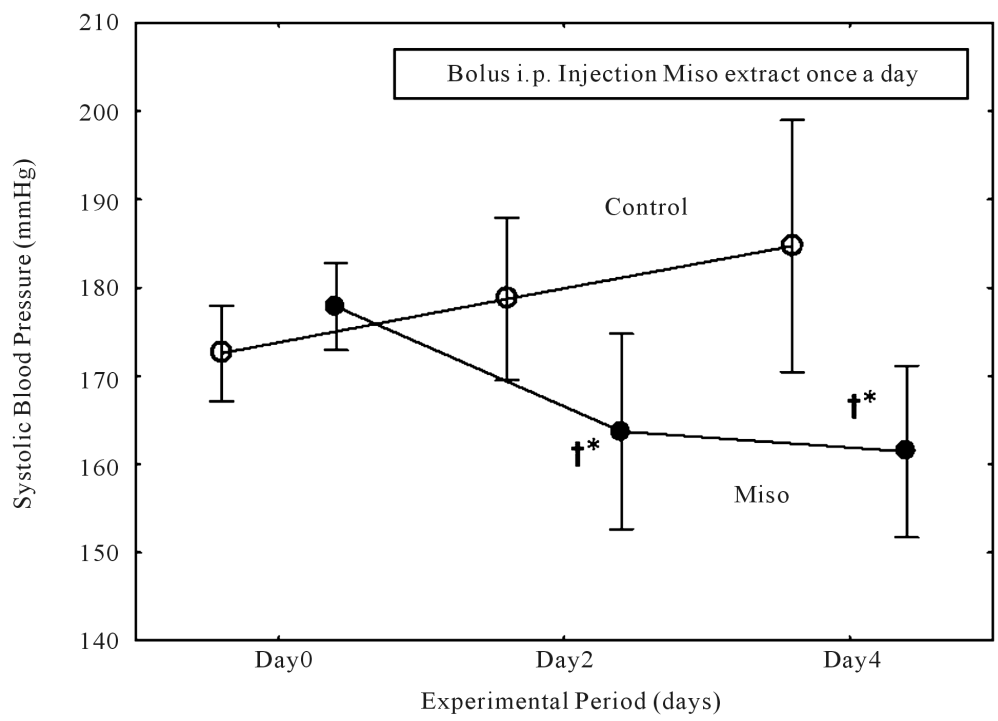

Figure 2. Changes in the SBP in response to an i.p. injection of the Miso extract for 4 days. Dahl salt-sensitive (Dahl S) rats fed a high-salt diet were given the Miso extract and the SBP changes were measured. The SBP was measured at before the injection (Day 0), after 2 days (Day 2) and 4 days (Day 4). The drawing module avoided the overlay of the SDs bars and the marks were described apart for each test period. Open circle, Dahl S rats given $0.5 \mathrm{~mL} 0.65 \%$ saline solution corresponding to the salt concentration in the Miso extract (Control); closed circle, Dahl S rats given $0.5 \mathrm{~mL}$ extract from $50 \mathrm{mg}$ Miso (Miso). * $\mathrm{p}<0.05$ vs value in $0.65 \%$ saline solution group, ${ }^{\dagger} \mathrm{p}<0.05$ vs the value before injection of the Miso extract (Day 0 ). Group differences were assessed by one-way factorial analysis of variance (ANOVA) followed by a post-hoc least significant difference (LSD) test.

The changes in SBP after administration of different doses of the Miso extract were shown in Figure 3 . The SBP was measured 4 hrs after the bolus i.p. injection of the Miso extract. The Miso extract decreased the SBP of 


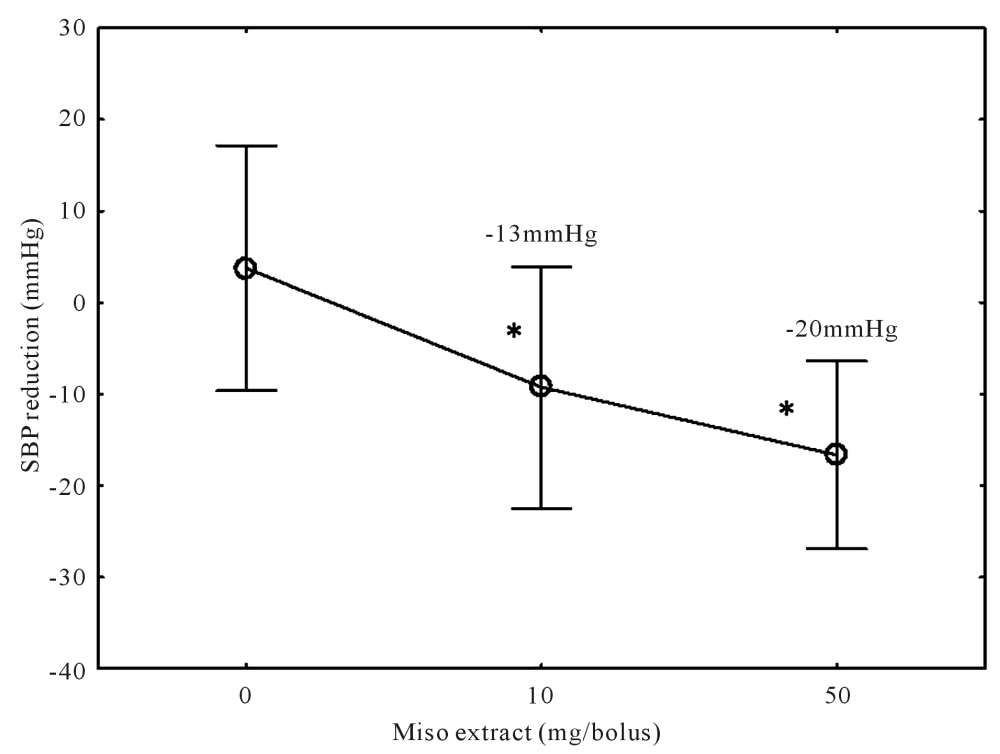

Figure 3. Dose-dependency of the Miso extract. The values are expressed as the difference between the SBP reduction in rats given the Miso extract and Dahl S rats given a saline solution. The marks showed the difference between BP in Dahl S rats that were injected Miso extract and that in each control (saline solution). The figures represented the difference from the zero Miso extract. * p < 0.05 vs value in Dahl S rats given a saline solution. Group differences are assessed by one-way factorial analysis of variance (ANOVA).

Dahl S rats in a dose-dependent manner.

The crude Miso extract was passed through the membrane sieves (cutoff point $<5 \mathrm{kDa}$ ) and we obtained $<5$ $\mathrm{kDa}$ fraction of the Miso extract. At 4 hrs after the i.p. injection, we measured and compared the antihypertensive activity between the crude ( $50 \mathrm{mg}$ Miso) and fractionated extract $(<5 \mathrm{kDa})$. There was no difference in the antihypertensive activity between the 2 samples, suggesting that the antihypertensive factor(s) was expected to be present in the $<5 \mathrm{kDa}$ fraction (Figure 4 ).

\subsection{Long-Term Subcutaneous Infusion of Miso Extract in Rats with Salt-Induced Hypertension}

We examined the long-term effects of the low-dose (50 mg/day) Miso extract on SBP in Dahl S rats with salt-induced hypertension using a continuous, osmotic mini-pump working for 14 days. As shown in Figure 5, there was no difference in the body weight between the DS-high and DS-miso groups.

Systolic blood pressure increased in a time-dependent manner in the DS-high group when compared with that in the DS-low group (Figure 6). In DS-miso, however, the SBP elevation was significantly attenuated at day 4 and day 14, as compared with that in the DS-high group (144 $\pm 4 \mathrm{mmHg}$ vs $167 \pm 6 \mathrm{mmHg}$ at day $4, \mathrm{p}<0.001$; $155 \pm 13 \mathrm{mmHg}$ vs $172 \pm 8 \mathrm{mmHg}$ at day $14, \mathrm{p}<0.005)$.

The heart weight of the DS-high group increased as compared with that of the DS-low group (Table 1 ). The increase in heart weight observed in the DS-high group was attenuated significantly in the DS-miso group. Similarly, the aortic weight increased in the DS-high group when compared with that in the DS-low group; this increase was attenuated with administration on the Miso extract. The difference, however, was not significant. The kidney weight was lower in the DS-miso group than in the DS-high group; additionally, the increase in urinary protein excretion in the hypertensive DS-high group was significantly decreased in the Dahl S rats that were given the Miso extract (Figure 7).

Next, to understand the role of the kidneys in sodium handling with respect to the reduction in blood pressure by the subcutaneous infusion of the Miso extract, we examined the parameters indicating renal sodium metabolism (Table 2). Urinary volume and urinary excretion of sodium significantly decreased in the DS-miso group compared with that in the DS-high group; neither the sodium clearance rate nor the fractional excretion of sodium (FENa) changed after the subcutaneous infusion of the Miso extract. These findings are supported by the fact that there were no differences in the osmotic clearance rate between the DS-high and DS-miso groups. On 


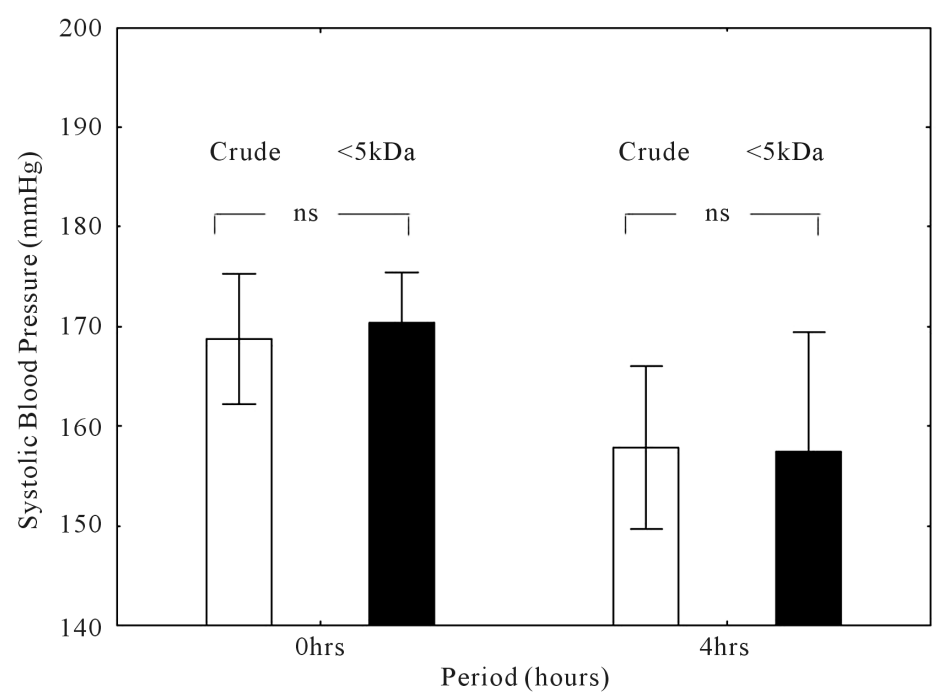

Figure 4. The hypotensive activity in the $<5 \mathrm{kDa}$ Miso extract. Crude, Miso extract; $<5 \mathrm{kDa}$, fractionated Miso extract using membrane sieves. $0 \mathrm{hr}$, SBP before the i.p. injection; 4 hrs, SBP 4 hrs after the i.p. injection. No differences in SBP were noted between the Dahl S rats given crude and those given $<5 \mathrm{kDa}$ Miso extract. ns represents statistically not significant.

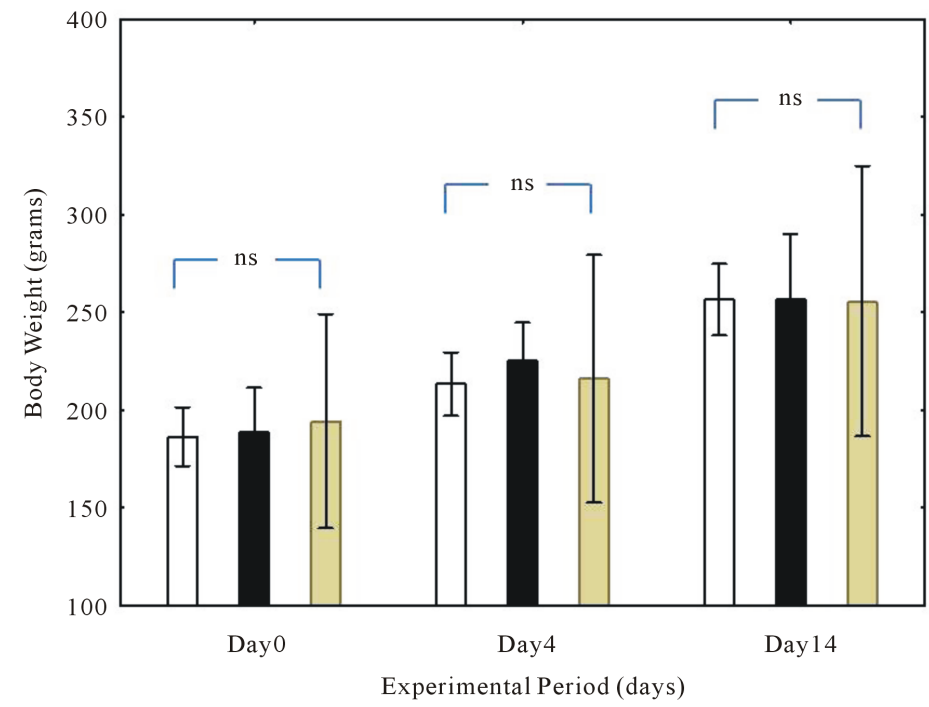

Figure 5. Body weights. Open column, control Dahl S rats with hypertension (DS-high); solid column, Dahl S rats given Miso extract (DS-miso); gray column, Dahl S rats fed a low-salt diet (DS-low). ns represents statistically not significant. Day 0 the data before the implantation of an osmotic mini-pump.

the other hand, free water clearance was significantly decreased in the DS-miso group compared with that in the DS-high group.

In addition, it is noted that $\mathrm{Na} / \mathrm{K}$ in urine was significantly lower in Dahl $\mathrm{S}$ rats fed a low-salt diet than in Dahl S rats fed a high-salt diet, suggesting that $\mathrm{Na} / \mathrm{K}$ exchange was enhanced in the low-salt Dahl S rats through up-regulated renin-angiotensin system on a low-salt diet. In DS-miso, however, the $\mathrm{Na} / \mathrm{K}$ balance was not changed when compared with DS-high.

\section{Discussion}

Fermented Miso is an integral part of Japanese cuisine. Miso usually contains 10\% - 13\% salt (w/w) and 10 - $30 \mathrm{~g}$ Miso/day is served as Miso soup. This means that the intake of salt from Miso averages $1-3.9 \mathrm{~g} /$ day, because of which, in Japan, Miso is believed to be responsible for incidence of hypertension. To our knowledge, however, 


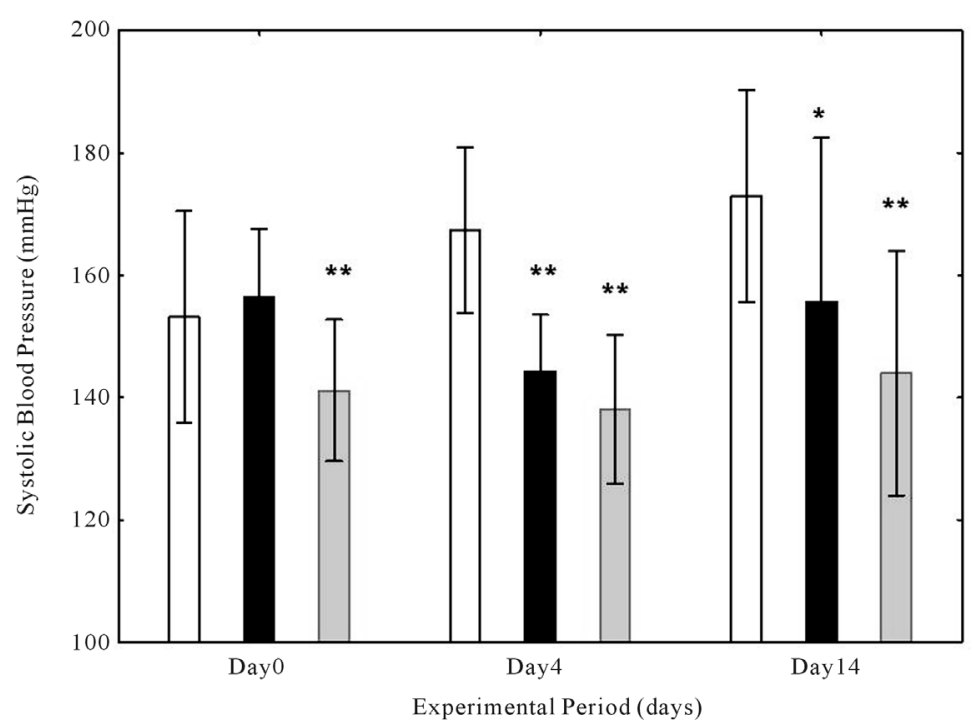

Figure 6. Systolic blood pressures in Dahl S rats. Open column, control Dahl S rats with hypertension (DS-high); solid column, Dahl S rats given the Miso extract (DS-miso); gray column, Dahl S rats fed a low-salt diet (DS-low). ns represents statistically not significant. Day 0 , the data before the implantation of an osmotic mini-pump. ${ }^{*} \mathrm{p}<0.05,{ }^{* *} \mathrm{p}<0.001$ vs the DS-high group (open column). Group differences were assessed by one-way factorial analysis of variance (ANOVA) followed by a post-hoc least significant difference (LSD) test.

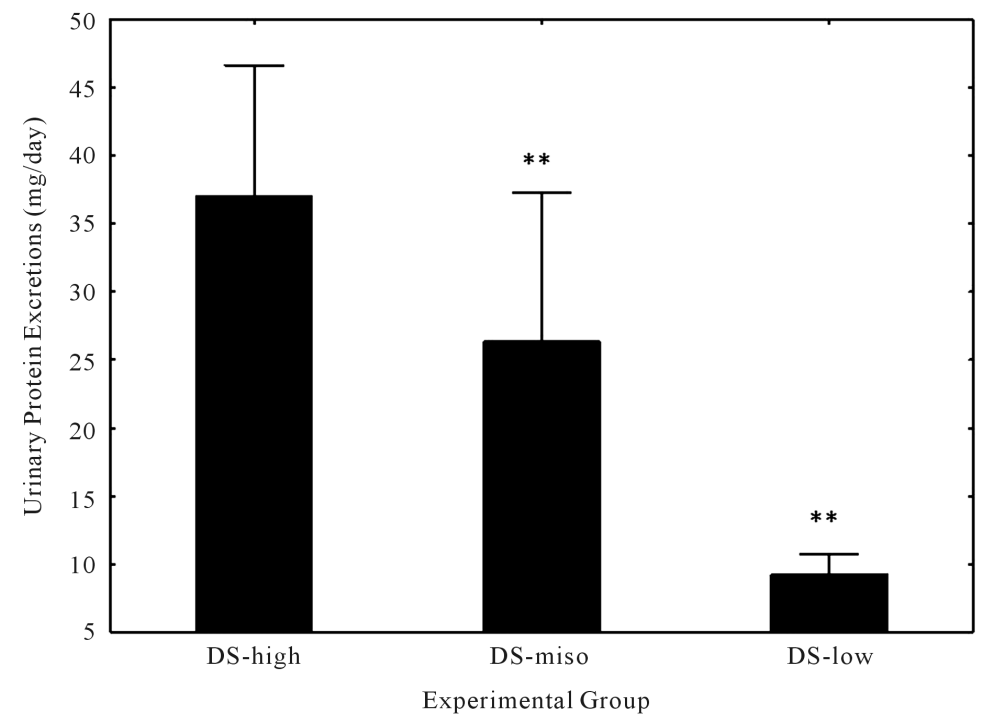

Figure 7. The effects of Miso extract on urinary protein excretion. DS-high, Dahl S rats fed a high-salt diet; DS-miso, Dahl $\mathrm{S}$ rats fed a high-salt diet and given Miso extract; DS-low, Dahl S rats fed a low-salt diet. Group differences were assessed by one-way factorial analysis of variance (ANOVA) followed by post-hoc least significant difference (LSD) test. ${ }^{* *} \mathrm{p}<001$ vs DS-high.

there are no studies showing that the intake of Miso soup induces hypertension in humans. Our preliminary, cross-sectional survey on subjects at an annual medical checkup found that the more frequent intake of Miso soup consuming 3.0 - 4.5 g salt is not associated with an elevation of blood pressure, compared with less than once a day intake of Miso soup consuming less than $1.5 \mathrm{~g} / \mathrm{day}$, suggesting that Miso contains some nutrients that attenuates blood pressure elevation due to higher salt intake (unpublished data).

To test the possibility that Miso contributes to hypertension, we examined the influence of long-term oral intake of Miso soup in salt-induced hypertension of Dahl S rats. We found that long-term intake of Miso prevents salt-induced hypertension as well as attenuates heart and kidney damage [1]. The reduction in blood pressure by 
Table 1. The Effects of subcutaneous infusion of Miso extracts on organ weights.

\begin{tabular}{ccccc}
\hline Organ & Unit & DS-high & DS-miso & DS-low \\
\hline Heart weight & $\mathrm{g} / 100 \mathrm{~g} \mathrm{BW}$ & $0.450 \pm 0.025$ & $0.392 \pm 0.022^{*}$ & $0.420 \pm 0.106$ \\
Aortic weight & $\mathrm{mg} / \mathrm{mm}^{2}$ & $0.327 \pm 0.078$ & $0.293 \pm 0.036$ & $0.260 \pm 0.025^{*}$ \\
Kidney weight & $\mathrm{g} / 100 \mathrm{BW}$ & $0.592 \pm 0.056$ & $0.550 \pm 0.013^{*}$ & $0.468 \pm 0.015^{* *}$ \\
\hline
\end{tabular}

${ }^{*} \mathrm{p}<0.05,{ }^{* *} \mathrm{p}<0.001$ vs DS-high.

Table 2. The effects of subcutaneous infusion of Miso extracts on Na handling in the kidney.

\begin{tabular}{cccc}
\hline Variables & DS-high & DS-miso & DS-low \\
\hline $\mathrm{UV}$ & $14.47 \pm 1.46$ & $9.69 \pm 2.53^{* *}$ & $2.64 \pm 0.40^{* *}$ \\
$\mathrm{U}_{\mathrm{Na}} \mathrm{V}$ & $4.45 \pm 0.41$ & $3.81 \pm 0.99^{* * *}$ & $0.09 \pm 0.01^{* *}$ \\
$\mathrm{U}_{\mathrm{K}} \mathrm{V}$ & $1.10 \pm 0.14$ & $1.04 \pm 0.32$ & $0.90 \pm 0.20$ \\
$\mathrm{U}_{\mathrm{Na}} / \mathrm{U}_{\mathrm{k}}$ & $4.0 \pm 0.3$ & $3.7 \pm 0.6$ & $0.1 \pm 0.0^{* *}$ \\
$\mathrm{Ccr}$ & $1.74 \pm 0.18$ & $1.46 \pm 0.20^{*}$ & $1.00 \pm 0.22^{* *}$ \\
$\mathrm{C}_{\mathrm{Na}}$ & $30.55 \pm 3.18$ & $27.65 \pm 7.01$ & $0.74 \pm 0.10^{* *}$ \\
$\mathrm{FENa}$ & $1.76 \pm 0.24$ & $1.89 \pm 0.45$ & $0.07 \pm 0.01^{* *}$ \\
$\mathrm{P}_{\mathrm{Osm}}$ & $314 \pm 4$ & $310 \pm 2^{*}$ & $307 \pm 4^{* *}$ \\
$\mathrm{U}_{\mathrm{Osm}}$ & $15.3 \pm 1.3$ & $15.7 \pm 3.4$ & $6.3 \pm 0.9^{* *}$ \\
$\mathrm{C}_{\mathrm{Osm}}$ & $48.9 \pm 4.4$ & $50.6 \pm 11.0$ & $20.7 \pm 3.2^{* *}$ \\
$\mathrm{C}_{\mathrm{H}_{2} \mathrm{O}}$ & $-34.4 \pm 4.2$ & $-40.9 \pm 8.7^{*}$ & $-18.1 \pm 3.3^{* *}$ \\
\hline
\end{tabular}

$\mathrm{UV}$, urine volume (mL/100g BW/day); UNaV, urinary excretion of sodium (mEq/100g BW/day); UKV, urinary excretion of potassium (mEq/100g BW/day): UNa/Uk, ratio of urinary sodium/potassium; Ccr, creatinine clearance $(\mathrm{L} / 100 \mathrm{~g}$ BW/day); CNa, clearance of spdum (mL/100g BW/day); FENa, fractional excretion of sodium (\%); POsm, plasma osmolality $\left(\mathrm{mOsm} / \mathrm{KgH}_{2} \mathrm{O}\right)$; UOsm, urinary osmolality $\left(\mathrm{Osm} / \mathrm{KgH}_{2} \mathrm{O}\right)$; COsm, clearance of osmolality $\left(\mathrm{mOsm} / \mathrm{KgH}_{2} \mathrm{O} / 100 \mathrm{~g}\right.$ $\mathrm{BW} /$ day); $\mathrm{CH}_{2} \mathrm{O}$, free water clearance (mL/100g BW/day). p values indicate statistically significant between DS-high and DS-miso. ${ }^{*} \mathrm{p}<0.05,{ }^{* *} \mathrm{p}<0.001$ vs DS-high.

the oral intake of 5 - $10 \mathrm{~g}$ Miso/day was associated with enhanced natriuresis/diuresis, likely mediated by the urinary dopaminergic system [2].

Thus, the present study examined whether a Miso extract decreases blood pressure via the circulation. We demonstrated that a bolus i.p. injection of Miso extract decreases the SBP in salt-induced hypertension in Dahl S rats fed a high-salt diet. The finding suggests that the Miso extract contains a substance(s) that decreases SBP in Dahl S rats via the circulation. Furthermore, the antihypertensive substance(s) is heat-stable and less than $5 \mathrm{kDa}$ in molecular weight. These data suggested that there are several substances to decrease blood pressure in Miso, and we provided evidence for the occurrence of antihypertensive substance(s) that are different from the activity shown in the previous studies.

We administered $0.5 \mathrm{~mL}$ of the extract prepared using $50 \mathrm{mg}$ Miso (10\% Miso solution). The amount of Miso given via the i.p. route is much lower than that given via the oral route as reported previously [1] [2]. This suggests that mechanisms for these effects may be different depending on the route of administration. To test this hypothesis, we examined the long-term antihypertensive action of Miso using an osmotic mini-pump placed in the subcutaneous tissue and investigated the sodium handling by the kidney using a metabolic cage. In the study where the administration was via the subcutaneous route, we infused an extract of $50 \mathrm{mg}$ Miso/day. A continuous, 14-day subcutaneous infusion of $50 \mathrm{mg}$ Miso extract/day attenuated the blood pressure. The blood pressure reduction was associated with decreases in the heart weight or kidney weight and tended to be associated with a decrease in the aortic weight, suggesting that the antihypertension is associated with attenuation of cardiovascular damage. This is also supported by the finding that proteinuria was lower in the group given the Miso extract than in the control Dahl S rats that were fed a high-salt diet. 
Such a reduction in blood pressure was not accompanied by increases in natriuresis and diuresis that was observed for administration via the oral route. Additionally, neither osmotic nor free water clearance changed, suggesting that the Miso extract does not affect urinary excretion of solutes. These data indicate that the mechanism of antihypertension via the circulation is different from the mechanism via the oral route. The mechanism via the subcutaneous route is not clear; however, some reports indicate that peptides from soybeans may suppress the renin-angiotensin system, thereby decreasing the blood pressure [9]-[11]. In our preliminary study, however, we found that the antihypertensive substance(s) does not occur in the soybean homogenate per se and the activity was more marked in 2 - 3 months' fermented rather than 1 - 2 years' matured Miso (unpublished data). These might suggest that the substances may be peptides produced from soybeans by fermentation. It is unclear whether the Miso extract inhibits renin-angiotensin system. However, the $\mathrm{Na} / \mathrm{K}$ balance in urine was not altered in Dahl S rats given the Miso extract when compared with Dahl S rats with hypertension (Table 2). If renin-angiotensin system was inhibited by the Miso extract, the secretion of aldosterone would have declined and this decreases the aldosterone-sensitive $\mathrm{Na} / \mathrm{K}$ exchange in the distal tubules in the kidneys, thereby increasing the $\mathrm{Na} / \mathrm{K}$ balance in urine. We did not directly measure the status of renin-angiotensin system; however, these data appeared to be against suppression of renin-angiotensin system by the Miso extract. To address these points directly, we are required to investigate the pharmacological properties of the current antihypertensive activity in the Miso extract. We are under way to purify and identify the antihypertensive activity via the subcutaneous route.

We did not infuse the Miso extract directly into vascular vessels mainly because the influence by the operation was minimized. We believe that the direct determination of blood pressure was the next step in studying pharmacological properties of the antihypertensive activity along with purification and identification.

The Dietary Approaches to Stop Hypertension (DASH) diet has been reported to decrease the blood pressure compared with an American style diet [12] [13]. The DASH diet is rich in vegetables (plant fibers, potassium, and calcium) and low in fat. The blood pressure reduction noted with the DASH diet is independent of the salt-intake (intake range, 4 to $8 \mathrm{~g} /$ day), suggesting that blood pressure is related not only to salt-intake, but also to intake of various nutrients in a balanced manner. We believe that this is true for Miso extract as well, because some substances from fermented soybeans and molted rice attenuate salt dependent hypertension through several mechanisms.

\section{Conclusion}

In conclusion, the present study shows that treatment with very low doses of Miso extract via the i.p. or subcutaneous routes decreases blood pressure in rats with salt-induced hypertension. This blood pressure reduction is not mediated by increases in sodium excretion from the kidneys. The blood pressure reduction via the subcutaneous route is associated with the attenuation of cardiovascular stress following hypertension. Miso contains several substances beneficial for health [1]-[3] [14]-[18]. These factors involved in the antihypertensive effects of Miso through integrated mechanisms. However, further study is required to identify these substances.

\section{Acknowledgements}

We acknowledge Kyoko Nakano and Yoshihide Obata, Central Miso Research Institute, Tokyo, Japan for the gift of Miso. This study is supported in part by Grant-in-Aids from Center for Interdisciplinary Studies of Science and Culture, Kyoritsu Women's University \& Junior College (2012) and a grant from Central Miso Research Institute (2012).

\section{Declaration of Interest}

The authors report no conflicts of interest. The authors alone are responsible for the content and writing of the paper.

\section{References}

[1] Yoshinaga, M., Toda, N., Tamura, Y., Terakado, S., Ueno, M., Otsuka, K., Numabe, A., Kawabata, Y., Uehara, Y. (2012) Japanese Traditional Miso Soup Attenuates Salt-Induced Hypertension and Its Organ Damage in Dahl SaltSensitive Rats. Nutrition, 28, 924-931. http://dx.doi.org/10.1016/j.nut.2011.09.010 
[2] Du, D.D., Yoshinaga, M., Sonoda, M., Kawakubo, K. and Uehara, Y. (2013) Blood Pressure Reduction by Japanese Traditional Miso Is Associated with Increased Diuresis and Natriuresis through Dopamine System in Dahl Salt-Sensitive Rat. Clinical and Experimental Hypertension, 36, 359-366.

[3] Watanabe, H., Kashimoto, N., Kajimura, J. and Kamiya, K. (2006) A Miso (Japanese Soybean Paste) Diet Conferred Greater Protection against Hypertension than a Na Chloride Diet in Dahl Salt-Sensitive Rats. Hypertension Research, 29, 731-738. http://dx.doi.org/10.1291/hypres.29.731

[4] Ebisawa, K., Miyamoto, Y., Du, D.D., Yoshinga, M. and Uehara, Y. (2012) The Effects of Long-Term Miso Soup on $\mathrm{Na}$ Absorption in the Intestine of Wistar Rats. The 35th Scientific Meeting of Japanese Society of Hypertension, Nagoya, 20-22 September 2012.

[5] Friedman, M. and Freed, S.C. (1949) Microphonic Manometer for Indirect Determination of Systolic Blood Pressure in the Rat. Proceedings of the Society for Experimental Biology and Medicine, 70, 670-672. http://dx.doi.org/10.3181/00379727-70-17028

[6] Hirawa, N., Uehara, Y., Kawabata, Y., Ohshima, N., Ono, H., Nagata, T., Gomi, T., Ikeda, T., Goto, A., Yagi, S. and Omata, M. (1995) Subpressor Dose of Angiotensin II Increases Susceptibility to the Haemodynamic Injury of Blood Pressure in Dahl Salt-Sensitive Rats. Journal of Hypertension, 13, 81-90. http://dx.doi.org/10.1097/00004872-199501000-00013

[7] Uehara, Y., Numabe, A., Hirawa, N., Kawabata, Y., Iwai, J., Ono, H., Matsuoka, H., Takabatake, Y., Yagi, S. and Sugimoto, T. (1991) Antihypertensive Effects of Cicletanine and Renal Protection in Dahl Salt-Sensitive Rats. Journal of Hypertension, 9, 719-728. http://dx.doi.org/10.1097/00004872-199108000-00005

[8] Moriya, C., Shida, Y., Yamane, Y., Miyamoto, Y., Kimura, M., Huse, N., Ebisawa, K., Kameda, Y., Nishi, Y., Du, D.D., Yoshinaga, M., Murota, I., Sato, N. and Uehara, Y. (2013) Subcutaneous Administration of Sodium Alginate Oligosaccharides Prevents Salt-Induced Hypertension in Dahl Salt-Sensitive Rats. Clinical and Experimental Hypertension, 35, 607-613. http://dx.doi.org/10.3109/10641963.2013.776568

[9] Martinez-Villaluenga, C., Torino, M.I., Martín, V., Arroyo, R., Garcia-Mora, P., Estrella Pedrola, I., Vidal-Valverde, C., Rodriguez, J.M. and Frias, J. (2012) Multifunctional Properties of Soy Milk Fermented by Enterococcus faecium Strains Isolated from Raw Soy Milk. Journal of Agricultural and Food Chemistry, 60, 10235-10244. http://dx.doi.org/10.1021/jf302751m

[10] Tomatsu, M., Shimakage, A., Shinbo, M., Yamada, S. and Takahashi, S. (2013) Novel Angiotensin I-Converting Enzyme Inhibitory Peptides Derived from Soya Milk. Food Chemistry, 136, 612-616. http://dx.doi.org/10.1016/j.foodchem.2012.08.080

[11] Farzamirad, V. and Aluko, R.E. (2008) Angiotensin-Converting Enzyme Inhibition and Free-Radical Scavenging Properties of Cationic Peptides Derived from Soybean Protein Hydrolysates. International Journal of Food Sciences and Nutrition, 59, 428-437. http://dx.doi.org/10.1080/09637480701592897

[12] Appel, L.J., Moore, T.J., Obarzanek, E., William, M., Volomer, W.M., Svetkey, L.P., Sacks, F.M., Bray, G.A., Vogt, T.M., Cutler, J.A., Windhauser, M.M., Lin, P.-H. and Karanja, N., The DASH Collaborative Research Group (1997) A Clinical Trial of the Effects of Dietary Patterns on Blood Pressure. New England Journal of Medicine, 336, 1117-1124. http://dx.doi.org/10.1056/NEJM199704173361601

[13] Sacks, F.M., Svetkey, L.P., Vollmer, W.M., Appel, L.J., Bray, G.A., Harsha, D., Obarzanek, E., Conlin, P.R., Miller 3rd, E.R., Simons-Morton, D.G., Karanja, N. and Lin, P.H. (2001) DASH-Na Collaborative Research Group: Effects on Blood Pressure of Reduced Dietary Na and the Dietary Approaches to Stop Hypertension (DASH) Diet. DASH-Na Collaborative Research Group. New England Journal of Medicine, 344, 3-10. http://dx.doi.org/10.1056/NEJM200101043440101

[14] Watanabe, H. (2013) Beneficial Biological Effects of Miso with Reference to Radiation Injury, Cancer and Hypertension. Journal of Toxicologic Pathology, 26, 91-103. http://dx.doi.org/10.1293/tox.26.91

[15] Nevala, R., Vaskonen, T., Vehniainen, J., Korpela, R. and Vapaatalo, H. (2000) Soy Based Diet Attenuates the Development of Hypertension When Compared to Casein Based Diet in Spontaneously Hypertensive Rat. Life Science, 66, 115-124. http://dx.doi.org/10.1016/S0024-3205(99)00569-X

[16] He, J., Gu, D., Wu, X., Chen, J., Duan, X., Chen, J. and Whelton, P.K. (2005) Effect of Soybean Protein on Blood Pressure: A Randomized, Controlled Trial. Annals of Internal Medicine, 143, 1-9. http://dx.doi.org/10.7326/0003-4819-143-1-200507050-00004

[17] Velasques, M.T. and Bhathena, S.J. (2007) Role of Dietary Soy Protein in Obesity. International Journal of Medical Sciences, 4, 72-82. http://dx.doi.org/10.7150/ijms.4.72

[18] Known, D.Y., Hong, S.M., Ahn, I.I.S., Kim, M.J., Yang, H.J. and Park, S. (2011) Isoflavonoids and Peptides from Meju, Long-Term Fermented Soybeans, Increase Insulin Sensitivity and Exert Insulinotropic Effects in Vitro. Nutrition, 27, 244-252. http://dx.doi.org/10.1016/j.nut.2010.02.004 